\title{
Komplementsystem und Komplementdefekte
}

\author{
Michael Kirschfink
}

\section{$1 \quad$ Einleitung}

Unter den Mechanismen, die zum Selbstschutz des Körpers beitragen, ist das Komplementsystem von herausragender Bedeutung. Als Teil der angeborenen Abwehr ist es jederzeit verfügbar und somit bereits in der Präimmunphase, $d$. h. bevor es zur Bereitstellung spezifischer Antikörper und T-Lymphozyten kommt, von unschätzbarem Wert. Zu seinen Effektorfunktionen zählen neben der lytischen Zerstörung pathogener Mikroorganismen die Beseitigung von Immunkomplexen, die Opsonisierung von Krankheitserregern und von autoantigenem Material für eine effiziente Phagozytose sowie die Rekrutierung und Aktivierung von Entzündungszellen. Darüber hinaus moduliert das Komplementsystem aber auch die Antwort des erworbenen Immunsystems. Außerdem wird in zunehmendem Maße die Rolle des Komplementsystems in so unterschiedlichen biologischen Prozessen wie der Hämatopoese, Fortpflanzung und der Organregeneration deutlich, sodass das Konzept von Komplement als reinem Abwehrsystem erweitert werden muss zu einem mit vielen biologischen Funktionen vernetzten und steuernden System zur Aufrechterhaltung der Homöostase.

Etwa $5 \%$ aller Plasmaproteine sind Komplementfaktoren, die zum überwiegenden Teil in der Leber und durch Makrophagen synthetisiert werden. Aber auch Fibroblasten, Epithelzellen der Lunge und des Magen-Darmtraktes und weitere Gewebszellen, wie Astrozyten, Haut- und Muskelzellen, sind zur Synthese zumindest bestimmter Komplementproteine fähig. Aktuellen Erkenntnissen zufolge finden sich aber auch im Zellplasma von Abwehrzellen Komplementproteine und -rezeptoren, deren Funktion und Bedeutung noch nicht geklärt ist.

Die chromosomale Zuordnung der Komplementproteine ist in Tab. 1 dargestellt, wobei bestimmte Anhäufungen (Cluster) von Genen für funktionsähnliche Komponenten zu

M. Kirschfink ( $\bowtie)$

Universitätsklinikum Heidelberg, Institut für Immunologie, Heidelberg, Deutschland

E-Mail: michael.kirschfink@urz.uni-heidelberg.de erkennen sind. Einige Komplementgene (für C2, C4, Faktor B) liegen als sog. HLA-Klasse-III-Gene auf dem humanen Chromosom 6, dem Träger des Haupthistokompatibilitätskomplexes (MHC) in enger Nachbarschaft zu anderen wichtigen Genen, die für Proteine der Immunabwehr kodieren.

\section{$2 \quad$ Aktivierung des Komplementsystems}

Das Komplementsystem wird im Wesentlichen über 3 Hauptwege, den klassischen, den alternativen und den Lektin-Weg, in Form einer Kaskade aktiviert (Abb. 1). Einzelne Komponenten, wie C3 oder C5, können aber auch direkt durch Proteasen anderer Mediatorsysteme, wie dem Gerinnungsund Fibrinolysesystem, in biologisch aktive Peptide gespalten werden.

Jeder dieser auf unterschiedliche Weise initiierten Aktivierungswege mündet in eine gemeinsame Endstrecke, die mit der Bildung der sogenannten C3-Konvertase beginnt und über Zwischenstufen zum lytischen Membranangriffskomplex (C5b-9, membrane-attack complex, MAC) führt (Abb. 1).

\subsection{Klassischer Weg}

Der klassische Weg wird überwiegend durch Bindung von C1q an Antigen-Antikörper-Komplexe, die IgG oder IgM enthalten, aktiviert. Nach Antigenbindung kommt es zu einer Konformationsänderung in den Fc-Regionen der Immunglobuline, wodurch die komplementbindenden Bereiche für $\mathrm{Clq}$ zugänglich werden. Über mehrere enzymatische Reaktionen, an denen $\mathrm{C} 1 \mathrm{r}$ und $\mathrm{C} 1 \mathrm{~s}$ beteiligt sind, wird die C3-Konvertase aus der 4. und 2. Komponente (C4b2a) gebildet, die bis $\mathrm{zu}$ 1000 C3-Moleküle in C3a und C3b spalten kann. Eine Aktivierung des klassischen Weges ist antikörperunabhängig auch über CRP, gramnegative Bakterien, apoptotische Zellen und bestimmte virale Glykoproteine möglich. 
Tab. 1 Komplementdefekte (modifiziert nach Picard et al. 2018; Lopez-Lera et al. 2018; Skattum et al. 2011; Degn et al. 2011; Pettigrew et al. 2009; Wahn und Späth 2008)

\begin{tabular}{|c|c|c|c|}
\hline Komplementdefekt & Genlokalisation & $\begin{array}{l}\text { Publizierte Fälle } \\
\text { (n)/Frequenz }\end{array}$ & Assoziierte Symptome/Krankheitsbilder \\
\hline \multicolumn{4}{|l|}{ Komponenten } \\
\hline $\mathrm{C} 1 \mathrm{q}$ & $1 \mathrm{p} 36$ & $>40$ & SLE-ähnlich (>90\%, oft schwere Verlaufsformen), Infektionen \\
\hline $\mathrm{C} 1 \mathrm{r}$ & $12 \mathrm{p} 13$ & $<20$ & SLE-ähnlich, RA, Infektionen \\
\hline $\mathrm{C} 1 \mathrm{~s}$ & $12 \mathrm{p} 13$ & $<20$ & SLE-ähnlich, RA, Infektionen \\
\hline $\mathrm{C} 4(\mathrm{C} 4 \mathrm{~A}, \mathrm{C} 4 \mathrm{~B})$ & $6 \mathrm{p} 21$ & $<30$ & $\begin{array}{l}\text { SLE-ähnlich, RA, Infektionen, homozygot: schwere Verlaufsform; heterozygot: } \\
\text { oft klinisch unauffällig }\end{array}$ \\
\hline $\mathrm{C} 2$ & $6 \mathrm{p} 21$ & $\begin{array}{l}1: 10.000 \text { bis } \\
1: 20.000\end{array}$ & SLE-ähnlich, RA, Infektionen (Pneumonie), Vaskulitis, oft klinisch unauffällig \\
\hline $\mathrm{C} 3$ & $19 \mathrm{p} 13$ & $<30$ & Pyogene Infekte \\
\hline $\mathrm{C} 5$ & $9 q 33-$ & 40 & Neisserieninfekte (Meningitis), SLE \\
\hline C6 & $5 \mathrm{p} 13$ & $>50$ & Neisserieninfekte (Meningitis), SLE \\
\hline $\mathrm{C} 7$ & $5 \mathrm{p} 13$ & $>50$ & Neisserieninfekte (Meningitis), SLE \\
\hline $\mathrm{C} 8 \alpha-\gamma^{*} / \mathrm{C} 8 \beta$ & $\begin{array}{l}\mathrm{C} 8 \alpha / \beta: 1 \mathrm{p} 32 \\
\mathrm{C} 8 \gamma: 9 \mathrm{q} 34\end{array}$ & $\begin{array}{l}>50 \\
\text { (überwiegend } \\
\text { C } 8 \beta \text { ) }\end{array}$ & Neisserieninfekte (Meningitis), SLE \\
\hline C9 & $5 \mathrm{p} 14-$ & $\begin{array}{l}1: 1000 \text { (Japan), } \\
\text { sonst selten }\end{array}$ & Neisserieninfekte (meist asymptomatisch) \\
\hline Factor B & $6 \mathrm{p} 21$ & 1 & $\begin{array}{l}\text { Neisserieninfekte } \\
\text { aHUS (Gain-of-function-Mutationen) }\end{array}$ \\
\hline Factor D & $19 \mathrm{p} 13$ & $<10$ & Neisserieninfekte \\
\hline MBL & $10 \mathrm{q} 11$ & $\begin{array}{l}5-7 \% \\
\text { (Kaukasier) }\end{array}$ & Bakterielle Infekte (meist asymptomatisch) \\
\hline MASP1 & $3 \mathrm{q} 27$ & sehr selten & 3MC-Syndrom (Carnevale, Mingarelli, Malpuech and Michels syndrome) \\
\hline $\begin{array}{l}\text { Ficolin } \\
3 \text { (H-Ficolin) }\end{array}$ & $1 \mathrm{p} 36$ & 6 & Respiratorische Infekte, nekrotisierende Enterokolitis \\
\hline MASP2 & $1 \mathrm{p} 36$ & $\begin{array}{l}3-4 \% \\
\text { (Kaukasier) }\end{array}$ & Respiratorische Infekte, pyogene Infekte (meist asymptomatisch) \\
\hline \multicolumn{4}{|l|}{ Regulatoren } \\
\hline C1-Inhibitor & $11 \mathrm{q} 11-\mathrm{q} 13$ & $1: 50.000$ & Hereditäres Angioödem \\
\hline $\begin{array}{l}\text { C4-bindendes } \\
\text { Protein }\end{array}$ & $1 \mathrm{q} 32$ & $<10$ & Atypischer Morbus Behçet, Angioödem \\
\hline Properdin & Xp11 & $>100$ & Neisserieninfekte (Meningitis) \\
\hline Faktor H & $1 \mathrm{q} 32$ & $\begin{array}{l}<30 \\
\text { (homozygot) } \\
>100 \\
\text { (heterozygot) }\end{array}$ & $\begin{array}{l}\text { Infektionen, aHUS } \\
\text { aHUS, MPGN }\end{array}$ \\
\hline FHR1-5 & $1 q 32(1 q 32)$ & $\begin{array}{l}>100(5 \% \text { der } \\
\text { Kaukasier })\end{array}$ & $\begin{array}{l}\text { aHUS, RA, SLE } \\
\text { FHR-1-Defekt, häufig assoziiert mit Anti-Faktor-H-Autoantikörpern = sog. } \\
\text { DEAP (deficiency of CFHR-proteins and CFH-autoantibody positive) }\end{array}$ \\
\hline \multirow[t]{2}{*}{ Faktor I } & $4 q 25$ & $\begin{array}{l}>30 \\
\text { (homozygot) } \\
>50 \\
\text { (heterozygot) }\end{array}$ & $\begin{array}{l}\text { Infektionen (Sepsis, Meningitis, Pneumonie) } \\
\text { aHUS }\end{array}$ \\
\hline & $20 \mathrm{p} 11$ & $>30$ & aHUS \\
\hline \multicolumn{4}{|l|}{ Thrombomodulin } \\
\hline $\mathrm{CD} 46 / \mathrm{MCP}$ & $1 \mathrm{q} 32$ & $\begin{array}{l}>50 \\
\text { (überwiegend } \\
\text { heterozygot) }\end{array}$ & aHUS \\
\hline \multirow[t]{2}{*}{ CD55/DAF } & $1 \mathrm{q} 32$ & 11 & $\begin{array}{l}\text { Proteinverlustenteropathie, intestinale Lymphangiektasie, Thrombosen } \\
\text { (CHAPLE }{ }^{* * *} \text {-syndrome) }\end{array}$ \\
\hline & PIG-A: X & $1: 100.000$ & $\begin{array}{l}\text { Paroxysmale nächtliche Hämoglobinurie (somatische Mutation des } P I G-A \text { - } \\
\text { Gens }^{* *} \text { ) }\end{array}$ \\
\hline \multirow[t]{2}{*}{ CD59 } & $11 \mathrm{p} 13$ & 13 & Polyradikuloneuropathie, Schlaganfälle, Hämolyse \\
\hline & PIG-A: X & $1: 100.000$ & $\begin{array}{l}\text { Paroxysmale nächtliche Hämoglobinurie (somatische Mutation des } P I G-A \text { - } \\
\text { Gens**) }\end{array}$ \\
\hline
\end{tabular}


Tab. 1 (Fortsetzung)

\begin{tabular}{|c|c|c|c|}
\hline Komplementdefekt & Genlokalisation & $\begin{array}{l}\text { Publizierte Fälle } \\
\text { (n)/Frequenz }\end{array}$ & Assoziierte Symptome/Krankheitsbilder \\
\hline \multicolumn{4}{|l|}{ Rezeptoren } \\
\hline $\begin{array}{l}\text { CR3 (CD18/ } \\
\text { CD11b) } \\
\text { CR4 (CD18/ } \\
\text { CD11c, LFA-1) }\end{array}$ & $\begin{array}{l}\text { CD18: 21q22 } \\
\text { CD11b: 16p11 } \\
\text { CD11c: 16p11 }\end{array}$ & 1:1 Mio. & Leukozytenadhäsionsdefekt (LAD) \\
\hline $\begin{array}{l}{ }^{*} \text { Immer als } \mathrm{C} 8 \alpha-\gamma-\mathrm{D} \\
{ }^{* *} \text { Das PIG-A-Gen k } \\
\text { Ankers vieler Memb } \\
{ }^{* * *} \text { CHAPLE, Hyper } \\
\text { SLE systemischer Lu } \\
\text { rative Glomerulonep }\end{array}$ & $\begin{array}{l}\text { fekt, da die } \gamma \text {-Kett } \\
\text { diert für das Enzy } \\
\text { nmoleküle, wie C } \\
\text { tivation of comple } \\
\text { us erythematodes, } \\
\text { itis }\end{array}$ & $\begin{array}{l}\text { normalerweise ko } \\
\mathrm{n} \text { N-Acetylglukos } \\
55 \text { und CD } 59 \text {, ben } \\
\text { nent, angiaopathic } \\
R A \text { rheumatoide A }\end{array}$ & $\begin{array}{l}\text { ent an die } \alpha \text {-Kette gebunden wird. Das } \gamma \text {-Gen selbst ist intakt } \\
\text { inyltransferase, welches für die Bildung des Glukosylphosphatidylinositol(GPI)- } \\
\text { gt wird } \\
\text { ombosis, protein-losing enteropathy } \\
\text { itis, aHUS atypisches hämolytisch-urämisches Syndrom, MPGN membranprolife- }\end{array}$ \\
\hline
\end{tabular}

Abb. 1 Vereinfachte Darstellung der 3 Komplementaktivierungswege und deren Regulation (oval/ blau $=$ lösliche Regulatoren, rechteckig/weiß = membranständige Regulatoren; $S C P N$ Serum Carboxypeptidase N)

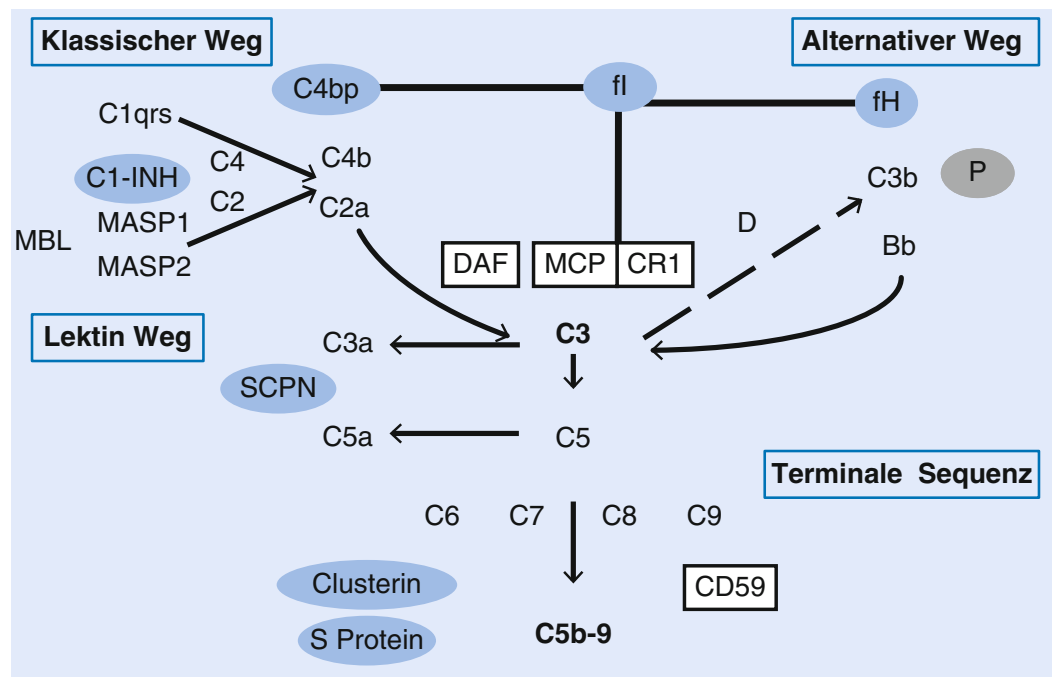

\subsection{Alternativer Weg}

Der alternative Weg wird bevorzugt von Mikroorganismen initiiert, kann aber auch durch ischämisch geschädigtes Gewebe und Fremdoberflächen, wie Membranen von Dialysegeräten oder während der extrakorporalen Oxygenierung aktiviert werden. Ausgangssituation ist die auf niedrigem Niveau ständig ablaufende spontane Hydrolyse der inneren Thioester-Bindung von $\mathrm{C} 3 \mathrm{zu} \mathrm{C} 3\left(\mathrm{H}_{2} \mathrm{O}\right)$, welche auch als sog. Tickover-Mechanismus bezeichnet wird. Das C3b-ähnliche $\mathrm{C} 3\left(\mathrm{H}_{2} \mathrm{O}\right)$ bindet an Faktor B, der daraufhin von Faktor D zu $\mathrm{Bb}$ gespalten wird. Die nun gebildete Flüssigphasen-C3Konvertase, $\mathrm{C} 3\left(\mathrm{H}_{2} \mathrm{O}\right) \mathrm{Bb}$, wird durch das Regulatorprotein Properdin stabilisiert. Properdin ist aber als ,pattern recognition molecule" auch selbst in der Lage, pathogene oder apoptotische Zellen zu erkennen und über die Bindung von C3 die C3-Konvertase zu generieren. Das durch diese Konvertase frisch gespaltene $\mathrm{C} 3 \mathrm{~b}$ ist für kurze Zeit für Bakterien, Pilze, Viren oder Tumorzellen und auch für körpereigene Zellen bindungsfähig, wird dann jedoch zum großen Teil schnell inaktiviert. An das oberflächengebundene C3b lagert sich Faktor B an, der dann von Faktor D gespalten wird. Die
Reaktion resultiert in der Bildung einer nun membranständigen $\mathrm{C} 3$-Konvertase (C3bBb).

\subsection{Lektin-Weg}

Der zuletzt entdeckte Lektin-Weg hat als Ausgangspunkt die Bindung des Mannose-bindenden Lektins (MBL) an terminale Zuckergruppen auf der Bakterienoberfläche. Diese Kohlenhydratgruppen werden als bakterienspezifische Muster, sog. pathogen associated molecular patterns (PAMPs), erkannt. MBL-assoziierte Serinproteasen (MASP) führen über die Spaltung von $\mathrm{C} 2$ und $\mathrm{C} 4$ zur Bildung der $\mathrm{C} 3$-Konvertase $\mathrm{C} 4 \mathrm{~b} 2 \mathrm{~b}$. Wie beim alternativen Aktivierungsweg ist der Lektin-Weg unabhängig von einer vorhergehenden Antikörperantwort und gehört somit zu den frühesten Abwehrmechanismen bei einer Infektion. Im Rahmen eines Ischämie-Reperfusions-Schadens wird der Aktivierung des Lektin-Wegs durch Glykosamine, mitochondriale Proteine und Phospholipide eine besondere Rolle zugesprochen. Ansonsten werden Defekte des Lektin-Wegs zumeist erst bei weiteren Immundysfunktionen klinisch auffällig. 
Alle 3 Komplementaktivierungswege haben nach der C3-Aktivierung eine gemeinsame Endstrecke, die mit der Bildung des lytischen Membranangriffskomplexes (MAC) abschließt. Dieser setzt sich zusammen aus je einem Molekül C5b, C6, C7, C8 und bis zu 18 Molekülen C9 und hat die Form eines Kanals, der sich in die Lipiddoppelschicht der Zellmembran einlagert. Durch nachfolgende Ionenströme wird die Zellfunktion eingeschränkt und bei genügend hohem Kalziumeinfluss erlischt schließlich die Funktion der Mitochondrien. Wenn eine ausreichende Anzahl an Poren an der Zellmembran gebildet wird, geht die Zelle durch Lyse zugrunde. Für Erythrozyten ist die Bildung von nur einer Pore schon ausreichend, um die Zellintegrität zu zerstören, was sich u. a. in Erkrankungen wie der paroxysmalen nächtlichen Hämoglobinurie (PNH) widerspiegelt (Abschn. 5.3).

Die nach Spaltung der dritten bzw. fünften Komponente freigesetzten Anaphylatoxine C3a und C5a zählen zu den stärksten Entzündungsmediatoren. Sie erhöhen die Permeabilität der Blutgefäße, induzieren die Kontraktion glatter Muskulatur und führen zur Produktion von Sauerstoffradikalen durch Makrophagen, neutrophilen und eosinophilen Granulozyten. Basophile Granulozyten und Mastzellen reagieren mit der Freisetzung von Histamin (pseudoallergische Reaktion). Diese Anaphylatoxine modulieren zudem die Synthese von IL-6, TNF- $\alpha$ und anderer Zytokine durch B-Lymphozyten und Monozyten und führen zur Rekrutierung verschiedener Abwehrzellen an den Infektionsort. Anaphylatoxine spielen durch ihre Wirkung auf inflammatorische Zellen in der Infektabwehr eine zentrale Rolle, sind aber auch wesentlich an der Pathogenese verschiedener Erkrankungen beteiligt (siehe unten). Interessanterweise wurden Anaphylatoxinrezeptoren auch auf Zellen parenchymatöser Organe wie Leber, Niere oder ZNS entdeckt, sodass eine systemübergreifende Wirkung dieser Peptide angenommen werden muss.

$\mathrm{C} 3 \mathrm{~b}$ opsonisiert Pathogene und Immunkomplexe für eine effiziente, durch Komplementrezeptor 1 (CR1, CD35) vermittelte Phagozytose und beteiligt sich durch Anlagerung an eine bereits bestehende $\mathrm{C} 3$-Konvertase an der Bildung der C5-Konvertasen $\mathrm{C} 4 \mathrm{~b} 2 \mathrm{a} 3 \mathrm{~b}$ oder $\mathrm{C} 3 \mathrm{~b}_{2} \mathrm{Bb}$. Das nach $\mathrm{C} 3 \mathrm{~b}$-Bindung an Antigen durch enzymatischen Abbau verbleibende C3dg/C3d führt durch Bindung an den Komplementrezeptor 2 (CR2, CD21) zur Herabsetzung der Aktivierungsschwelle von B-Zellen. Der zu den $\beta 2-$ Integrinen zählende Komplementrezeptor 3 (CR3, CD11b/ CD18) ist während der Leukozytenadhäsion und -transmigration sowie bei der Eliminierung von mit $\mathrm{iC} 3 \mathrm{~b}$, einem weiteren C3-Metaboliten, opsonisierten Partikeln von Bedeutung. In ähnlicher Weise stimuliert CR4 (CD11c/CD18) die Phagozytose.

\section{3}

Regulation des Komplementsystems

Eine Vielzahl an löslichen und membrangebundenen Komplementregulatoren greift in die Aktivierung des Komplementsystems ein (Abb. 1). Diese Regulatoren wirken als Inhibitoren der C3-Konvertase oder hemmen die Bildung des MAC.

$\mathrm{Zu}$ den löslichen Regulatoren der Komplementaktivierung zählen der C1-Inhibitor (C1-INH), Faktor H (fH), das C4-binding protein (C4bp), Faktor I (fI) und Properdin (P). Auf körpereigenen Zellen wird eine potenziell schädliche Komplementaktivierung durch die Oberflächenregulatoren CR1 (neben dessen Rezeptorfunktion), membrane cofactor protein (MCP/CD46) und decay accelerating factor (DAF/CD55) begrenzt.

Der C1-INH verhindert die Aktivierung des $\mathrm{C} 1$ - und des MBL/MASP-Komplexes. Ein Mangel an diesem, auch im Gerinnungs-, Fibrinolyse- und Kininsystem wirksamen multifunktionalen Regulator führt über die vermehrte Freisetzung von Bradykinin zum Angioödem (Abschn. 5.2). Faktor $\mathrm{H}$ fördert den spontanen Zerfall (,decay“) der C3Konvertase, $\mathrm{C} 3 \mathrm{bBb}$, und fungiert wie $\mathrm{MCP}$ und $\mathrm{CR} 1$ als Kofaktor bei der C3b-Inaktivierung durch Faktor I. C4bp übernimmt diese Kofaktorfunktion zur Regulation der klassischen C3-Konvertase, C4b2a. Properdin ist der einzig bekannte Regulator mit verstärkender Wirkung auf die Komplementaktivierung. Es stabilisiert die C3-Konvertase des alternativen Wegs. Durch seine kürzlich nachgewiesene Bindungsfähigkeit für Mikroorganismen und apoptotische Zellen ist Properdin auch in der Lage, den Alternativweg zu aktivieren.

DAF (CD55) ist ein Glykosyl-Phosphatidyl-Inositol(GPI)verankertes Membranprotein, das an $\mathrm{C} 3 \mathrm{~b}$ und $\mathrm{C} 4 \mathrm{~b}$ bindet und die Dissoziation des $\mathrm{Bb}$ von $\mathrm{C} 3 \mathrm{~b}$ und des $\mathrm{C} 2 \mathrm{~b}$ von $\mathrm{C} 4 \mathrm{~b}$ beschleunigt. MCP (CD46) ist ein membranständiges Glykoprotein, das die durch Faktor I vermittelte Inaktivierung von $\mathrm{C} 3 \mathrm{~b}$ fördert.

Die Bildung des MAC wird durch CD59, Clusterin und S-Protein/Vitronectin kontrolliert. CD59 ist wie CD55 ein GPI-gebundener Membranregulator, der an C8 und C9 bindet und dadurch die Bildung des MAC verhindert. S-Protein (Vitronectin) und Clusterin binden an C5b67 und verhindern deren Anlagerung an Zellmembranen. Der Nachweis von löslichen sC5b-9-Komplexen ist ein sensitiver Marker zur Abschätzung einer die gesamte Kaskade umfassenden Komplementaktivierung.

Anaphylatoxine werden durch die Carboxypeptidase N durch Abspaltung des C-terminalen Arginins inaktiviert $\left(\mathrm{C} 3 \mathrm{a}_{\text {desArg }}\right)$ oder stark in ihrer Aktivität eingeschränkt $\left(\mathrm{C} 5 \mathrm{a}_{\text {desArg }}\right)$.

Diese für den Schutz körpereigener Zellen so effektive Komplementregulation steht pathogenen Mikroorganismen in der Regel nicht zur Verfügung. Heute wissen wir aber, 
dass es vielen Erregern, wie Staphylokokken, Borrelien und HIV, gelingt, sich durch verschiedene Evasionsmechanismen dem Angriff des Komplementsystems zu entziehen. So nutzt das Epstein-Barr-Virus (EBV) CR2 als Eintrittspforte für die B-Zellinfektion. Serumresistente Borrelien entgehen dem Komplementangriff durch Bindung von Faktor $\mathrm{H}$ an spezifische Oberflächenproteine.

\section{$4 \quad$ Klinische Bedeutung des Komplementsystems}

Die pathophysiologische Bedeutung von Komplementstörungen liegt nicht nur in den relativ selten vorkommenden Komplementdefekten, sondern mehr noch in den Konsequenzen eines überaktivierten und/oder dysregulierten Systems, welche die Basis für eine Vielzahl von zum Teil schwerwiegenden Entzündungserkrankungen bilden. So ist Komplement beteiligt an der Entwicklung lebensgefährlicher Zustände, wie dem adult respiratory distress syndrome (ARDS), dem systemic inflammatory response syndrome (SIRS), der Sepsis und dem Multiorganversagen, z. B. nach schweren Traumata, Verbrennungen oder Infektionen. Eine überschießende Komplementaktivierung ist an der Entwicklung der rheumatoiden Arthritis, verschiedenen Formen der Glomerulonephritis, der Entstehung des Ischämie-Reperfusions-Schadens und der Transplantatabstoßung ursächlich beteiligt. Darüber hinaus beeinflusst Komplement die Entwicklung neurodegenerativer Erkrankungen, wie multiple Sklerose, Morbus Alzheimer oder Guillain-Barré-Syndrom. Experimentelle, wie auch klinische Studien belegen, dass Komplement auch für das Vollbild der Entzündung im Rahmen von Allergie und Asthma von Bedeutung ist.

\section{Defekte des Komplementsystems}

Defekte im Komplementsystem haben - sozusagen als Experimente der Natur - in großem Ausmaß zur Aufklärung der Reaktionsmechanismen sowie der physiologischen und pathophysiologischen Bedeutung dieses Kaskadensystems beigetragen. Sie umfassen je nach nationaler Studie ca. 5-10\% aller primären Immundefekte und sind insbesondere beim Fehlen von $\mathrm{C} 3-\mathrm{C} 8$ mit rezidivierenden Infektionen verbunden. Die Vererbung erfolgt in der Regel autosomal-rezessiv (Ausnahme: Properdindefekt X-chromosomal, C1-INH-Defekte autosomaldominant). Heterozygote Träger bleiben meist klinisch unauffällig. Hereditäre Defekte lassen sich durch exakte Anamnese und Familienanalyse weiter abklären. Vollständige Defekte sind für praktisch alle Komplement- und Regulatorproteine mit Ausnahme der Serum-Carboxypeptidase N beschrieben. Sekundäre
Defizienzen werden durch entzündungsbedingten Komplementverbrauch, Autoantikörper (z. B. gegen C1q, C1-Inhibitor, Faktor $\mathrm{H}$ ), verminderte Synthese und/oder gesteigerten Katabolismus verursacht.

Auch bei Defekten verschiedener Regulatorproteine (Faktor H, Faktor I, Properdin) stehen bakterielle Infektionen im Vordergrund des Krankheitsgeschehens (Tab. 1). Sind die beiden Membranregulatoren DAF/CD55 und CD59 betroffen, so resultiert daraus das Bild der paroxysmalen nächtlichen Hämoglobinurie ( $\mathrm{PNH}, \mathrm{Abschn}$. 5.3).

Bei Patienten mit C5-C8-Defekten ist das Risiko einer Meningokokkenerkrankung gegenüber der Normalbevölkerung 1000- bis 10.000-fach erhöht (Tab. 1). Betroffen sind häufiger Jugendliche und Erwachsene und das Keimspektrum umfasst eher seltene Serogruppen (W-35, Y) Hier ist die Impfung gegen N. meningitidis mit einem tetravalenten Konjugatimpfstoff dringend empfohlen, wenngleich damit nicht alle krankheitsrelevanten Serotypen erfasst werden. Ein weiterer Defekt, der schon in partieller Ausprägung zu rezidivierenden Neisserieninfektionen führt, ist der des Regulators Properdin. Rezidivierenden bakteriellen Infektionen im Kleinkindalter kann auch ein Defekt des Mannose-bindenden Lektins (MBL-Defekt) oder der MBL-assozierten Serinprotease (MASP2) zugrunde liegen. Diese zu einem hohen Prozentsatz auftretenden Defekte von Proteinen des LektinWeges (definiert als Plasmaspiegel $<100 \mathrm{ng} / \mathrm{ml}$ ) sind aber zumeist ohne klinische Konsequenzen.

\subsection{Systemischer Lupus erythematosus}

Interessanterweise sind Defekte von Komponenten der klassischen Aktivierungssequenz (C1, C4, C2) oftmals mit Autoimmunprozessen assoziiert (vor allem dem systemischen Lupus erythematodes [SLE] und lupusähnlichen Krankheitsbildern). Es gibt, je nach betroffener Komponente, eine starke Assoziation von teilweise über $90 \%$ zwischen Defekten im klassischen Aktivierungsweg und der Entwicklung eines SLE. Besonders schwerwiegend verläuft diese Krankheit bei Clq-Defekten. Bemerkenswert in diesem Zusammenhang ist die Beobachtung, dass auch ein Mangel an C1-Inhibitor, der zu einer Aktivierung des klassischen Aktivierungswegs führt, die Wahrscheinlichkeit erhöht, an SLE zu erkranken. Hierfür scheint die Entwicklung einer erworbenen C4- und C2-Defizienz verantwortlich zu sein. Sowohl die mangelnde Entsorgung apoptotischen Zellmaterials als auch die starke Beeinträchtigung der Elimination von Immunkomplexen durch Opsonisierung bilden dafür die molekulare Grundlage. Autoantikörper gegen C1q, die bei ca. 1/3 der Patienten nachweisbar sind, sind von prognostischer Bedeutung. 


\section{2 \\ Hereditäres Angioödem durch C1-INH-Mangel (HAE-C1-INH)}

Der weitaus häufigste, klinisch relevante Defekt eines Komplementregulators betrifft den C1-INH. Der Mangel dieses multifunktionellen Regulators führt zeitweise zu vaskulären Permeabilitätsstörungen mit der Symptomatik eines Angioödems (Quincke-Ödems). Von der genetischen Form des C1-INHMangels, dem hereditären Angioödem durch C1-INH-Mangel (HAE-C1-INH), sind andere Formen der bradykininvermittelten Angioödeme abzugrenzen, wie das hereditäre Angioödem mit normalem C1-INH (HAE nC1-INH, bei einem Teil der Patienten genetisch verursacht durch Mutationen im FXII-Gen, in anderen Fällen durch eine Mutation im Plasminogen-Gen oder im Angiopoietin-1-Gen), das Angioödem durch ACE-Hemmer und das seltene erworbene Angioödem durch C1-INH-Mangel (AAE-C1-INH) sowie die histaminvermittelten Haut- und Schleimhautschwellungen infolge von Allergien oder bei idiopathischer Urtikaria. Das HAE-C1-INH ist durch akute, anfallsweise auftretende Schwellungen an Händen, Füßen und im Gesicht, im Gastrointestinaltrakt (Koliken) und - besonders gefährlich - an Larynx und Pharynx charakterisiert. Die 2-5 Tage dauernden rezidivierenden Schwellungen beginnen zumeist im 1. oder 2. Lebensjahrzehnt.

Beim HAE-C1-INH Typ I (HAE-1; ca. 85 \% der Fälle) findet man verminderte Plasmaspiegel des C1-INH wie auch des C4. Die Synthese eines dysfunktionellen Inhibitors führt zum HAE Typ II (HAE-2), bei dem der C1-INH-Plasmaspiegel normal oder erhöht, die Funktion jedoch deutlich reduziert ist.

Die Therapie des HAE-C1-INH unterscheidet sich grundlegend von der allergischer Angioödeme oder gar einer Urtikaria durch die fehlende Wirksamkeit von Kortikosteroiden und Antihistaminika.

Für die Behandlung akuter Angioödemattacken des HAE-C1INH sind in Deutschland zurzeit 4 Medikamente (die C1-INHKonzentrate Berinert ${ }^{\circledR}$ und Cinryze ${ }^{\circledR}$; der rekombinante humane C1-INH Ruconest ${ }^{\circledR}$ und der Bradykinin-B2-Rezeptor-Antagonist Firazyr $^{\circledR}$ ) zugelassen. Für Kinder und Jugendliche stehen gegenwärtig Berinert ${ }^{\circledR}$ sowie für Kindern ab 2 Jahren Cinryze ${ }^{\circledR}$ und Firazyr $^{\circledR}$ zur Verfügung. Das aus humanem Plasma gewonnene C1-Inhibitor-Konzentrat $\left(\right.$ Berinert $^{\circledR}$ ) wird seit über 30 Jahren erfolgreich in der Akuttherapie eingesetzt. Eine wirksame Therapie bildet auch der Einsatz von Icatibant $\left(\right.$ Firazyr $\left.^{\circledR}\right)$, das akute Attacken eines HAE-C1-INH rasch zum Abklingen bringt. Berinert ${ }^{\circledR}$, Cinryze ${ }^{\circledR}$ und Firazyr ${ }^{\circledR}$ sind EU-weit auch zur Selbstanwendung bei der Behandlung akuter Attacken zugelassen.

Darüber hinaus ist in schweren Fällen eines HAE-C1-INH auch eine Langzeitprophylaxe möglich. Diese erfolgt am häufigsten mit regelmäßigen Injektionen von C1-INH-Konzentrat. Zugelassen für diese Indikation ist Cinryze ${ }^{\circledR}$. Einige Patienten erhalten auch abgeschwächte synthetische Andro- gene (z. B. Danazol, Oxandrolon). Diese führen zu einer Synthesesteigerung des C1-Inhibitors, sodass in der Regel genügend Schutz gegen Angioödemanfälle erreicht wird. Unter Berücksichtigung möglicher Nebenwirkungen (z. B. Thrombosen) kann im pädiatrischen Bereich die Anwendung des Antifibrinolytikums Tranexamsäure in Erwägung gezogen werden.

Beim AAE-C1-INH zeigen sich eine verminderte Konzentration und Funktion des C1-INH und ein vermindertes C4. Bei einem Teil dieser Patienten ist zusätzlich noch $\mathrm{Clq}$ im Plasma vermindert. Autoantikörper gegen C1-INH können vorhanden sein. Besonders wichtig bei dieser erworbenen Krankheit ist, dass bei einigen Patienten ein malignes Lymphom oder eine andere B-Zell-Störung der Symptomatik zugrunde liegt. Bei positiver Familienanamnese sollten selbst asymptomatische Neugeborene (Nabelschnurblut) und Kinder baldmöglichst auf Plasmaspiegel von C1-INH (Protein und Funktion) sowie von $\mathrm{C} 4$ getestet werden, gegebenenfalls ergänzt durch eine molekulargenetische Abklärung.

\subsection{Paroxysmal nächtliche Hämoglobinurie}

Die paroxysmal nächtliche Hämoglobinurie $(\mathrm{PNH})$ ist eine erworbene klonale Erkrankung der Hämatopoese, bei der eine oder mehrere pluripotente Stammzellen einen charakteristischen Defekt Glycosylphosphatidylinositol(GPI)-verankerter Oberflächenmoleküle aufweisen. Besonders betroffen davon sind Erythrozyten, deren fehlende Komplementregulation zu einer erhöhten Empfindlichkeit für die lytische Zerstörung durch Komplement führt. Das Fehlen der Regulatoren CD55 und CD59 beruht auf einer Mutation des Gens für Phosphatidyl-Isonitol-Glykan-A (PIGA) in den multipotenten hämatopoietischen Stammzellen im Knochenmark. Die Diagnose wird mittels zytofluorometrischem Nachweis der fehlenden Regulatoren auf Erythrozyten, wichtiger noch auf neutrophilen Granulozyten gestellt. Mithilfe des Fluorochroms FLAER (fluorescent aerolysin) kann auf Granulozyten und Monozyten direkt das Fehlen des GPI-Ankers dargestellt werden. Komplikationen der PNH sind Thrombosen und Niereninsuffizienz. Eine wirkungsvolle Therapie ist die Erythrozytentransfusion, zunehmend auch die Behandlung mit einem die Komplementlyse unterbindenden Anti-C5-Antikörper (Eculizumab, Soliris $\left.^{\circledR}\right)$. Voraussetzung für die Behandlung mit Eculizumab ist die vorherige Schutzimpfung gegen Meningokokken mit einem tetravalenten Konjugatimpfstoff gegen die Serogruppen A, C, W-135, Y und Serogruppe B. Steht die aplastische Anämie im Vordergrund wird mit Immunsuppressiva (z. B. CsA, ATG), gegebenenfalls auch mittels allogener Stammzelltransplantation behandelt.

Isolierte CD55-Defekte sind mit dem Krankheitsbild der Early-Onset Protein Loss Enterophathy verbunden, wogegen isolierte CD59-Defekte neben der Hämolyse schwere neuro- 
logische Erscheinungen (Polyradikuloneuropathie, Schlaganfälle) nach sich ziehen.

\subsection{Atypisches hämolytisch-urämisches Syndrom}

Das zu den thrombotischen Mikroangiopathien zählende atypische hämolytisch-urämische Syndrom (aHUS, D(-)-HUS) wird charakterisiert durch die Trias mikroangiopathische, Coombs-negative hämolytische Anämie, Thrombozytopenie und akutes Nierenversagen bei Ausschluss eines ADAMTS13Defekts (,A disintegrin and metalloprotease with thrombospondin-1-like domains"-verminderten Aktivität von ADAMTS-13 kommt es zu hohen vWF-Spiegeln im Blutplasma und damit zu einer spontanen Thrombozytenaktivierung). Ca. $50 \%$ dieser Fälle sind mit zumeist heterozygoten Mutationen in Genen der Komplementregulatoren $\mathrm{fH}$, fI, MCP/CD46 und Thrombomodulin, seltener mit sog. Gain-of-function-Mutationen der Gene für C3 und Faktor B assoziert. Dabei ist $\mathrm{fH}$ zwar serologisch oftmals unverändert, seine Bindungsfähigkeit an Nierenzellen und glomeruläre Basalmembran aufgrund von Mutationen in den für die Membranbindung wichtigen SCR19/20-Bereichen des fH-Moleküls jedoch gestört. Daneben werden, meist in Assoziation mit Defekten sog. fH-verwandter Moleküle (z. B. FHR1, FHR3), bei ca. $10 \%$ der aHUS-Patienten Autoantikörper gegen $\mathrm{fH}$ (seltener gegen andere Komplementproteine) nachgewiesen, die die Bindungsfähigkeit des Regulators an Zellen verhindern können. Meist sind bei aHUS, aber auch bei der weitaus häufigeren infektiösen Form dieses Krankheitsbildes $(\mathrm{D}(+) \mathrm{HUS}$, STEC-HUS, EHEC-HUS), neben C3 die Komplementgesamtfunktionen (CH50, AH50) vermindert sowie die Spiegel der Komplementaktivierungsprodukte $\mathrm{C} 3 \mathrm{a} / \mathrm{C} 3 \mathrm{~d}$ und sC5b-9 erhöht. Neben der Untersuchung auf Autoantikörper gegen Faktor H ist, auch zur Abgrenzung zu anderen Formen der mikroangiopathischen Thrombopenien (z. B. TTP), die gleichzeitige molekulargenetische Analyse der Komplementregulatoren $\mathrm{fH}$, fI, CD46/MCP, von C3 und Faktor B, aber auch von Thrombomodulin und Diacyl-Glycerol Kinase- $\epsilon$ (DGKE) unabdingbar. Viele der bisher nachgewiesenen Mutationen führen zur lokalen Dysregulation des Komplementsystems in der Niere. Da die inhibitorische Funktion des $\mathrm{fH}$ in der flüssigen Phase aber meist erhalten ist, werden diese Mutationen durch die serologische Diagnostik alleine nicht erfasst.

Neben der Substitution von fH durch Plasmaaustausch wird seit vielen Jahren ein Anti-C5-Antiköper (Eculizumab) erfolgreich eingesetzt. Seine Wirksamkeit sollte aber durch eine begleitende Komplementanalytik auch biochemisch kontrolliert werden (empfohlene Parameter: CH50, C3, C3a oder C3d, sC5b-9). Als kurativer Ansatz und Ultima Ratio wurde die kombinierte Leber-Nieren-Transplantation beschrieben.

\subsection{C3-Glomerulopathien}

Als weitere mit Störungen des Komplementregulation assoziierte Nierenerkrankungen müssen C3-Glomerulopathien (C3G) erwähnt werden, bei denen, wenn auch in weitaus geringerem Maß, ebenfalls Mutationen in Genen für Faktor H, FHR und C3, wie auch Antikörper gegen Faktor $\mathrm{H}$ gefunden werden. Insbesondere bei der histologisch definierten dense deposit disease (DDD, früher MPGN Typ 2) steht eine Dysregulation des Komplementsystems im Plasma durch den die C3-Konvertase stabilisierenden Autoantikörper C3-Nephritisfaktor im Vordergrund. Dieser Autoantikörper sowie ein stark verminderter C3-Spiegel wird auch bei Patienten mit erworbener partieller Lipodystrophie beobachtet, von denen ca. $25 \%$ später eine $\mathrm{C} 3 \mathrm{G}$ entwickeln.

\subsection{Altersbedingte Makuladegeneration}

Die altersbedingte Makuladegeneration (AMD) als genetisch komplexe, multifaktorielle Erkrankung der Netzhaut und angrenzender Strukturen ist hierzulande die führende Ursache der Blindheit im höheren Alter. Die Bildung von sog. Drusen führt zu einem ausgeprägten zentralen Visusverlust. Vor allem Austausch von Thymin (T) durch ein Cytosin (C) im fH-Gen (Y402H-Polymorphismus) ist bereits im heterozygoten, mehr noch im homozygoten Zustand, mit einem deutlich erhöhten Erkrankungsrisiko vergesellschaftet. Weitere krankheitsrelevante Polymorphismen betreffen die Gene von $\mathrm{C} 2, \mathrm{C} 3$, Faktor B und FHR-1.

\subsection{Leukozyten-Adhäsionsdefekt 1}

Komplementrezeptoren befinden sich auf einer Vielzahl von Körperzellen. Defekte von CR1 sind oft mit dem Bild eines SLE assoziiert. Sind die zu den Adhäsionsmolekülen ( $\beta_{2^{-}}$ Integrine) zählenden Komplementrezeptoren CR3 und CR4 betroffen, so äußert sich diese Defizienz als LeukozytenAdhäsionsdefekt 1 (LAD-I) mit kaum beherrschbaren bakteriellen Infektionen. Dieser Defekt stellt eine lebensbedrohliche Beeinträchtigung der Leukozytenfunktion dar. Ein früher Hinweis auf diese Störung ist häufig eine verzögerte AbstoBung und Entzündung des Nabelschnurstumpfs des Neugeborenen. Aufgrund eines Defekts von Adhäsionsmolekülen können die Leukozyten nicht an die Gefäßwand binden, um ins Gewebe zu wandern. Ursache ist das Fehlen der gemeinsamen CD18-Untereinheit von CR3 (CD11b/CD18) und CR4 (CD11c/CD18). Eine Diagnosestellung kann mittels zytofluorometrischer Analyse des CD18-Moleküls erfolgen. Als Therapie ist vor allem in schweren Verlaufsformen eine Stammzelltransplantation indiziert. 


\section{Diagnostik des Komplementsystems}

Die moderne Komplementanalytik geht weit über die traditionellen Parameter $\mathrm{C} 3$ und $\mathrm{C} 4$ hinaus, die nur in wenigen Fällen eine Aussage über eine Defizienz, eine Funktionseinschränkung oder gar den Aktivierungszustand dieses komplexen Systems erlauben. Die bisher nur in wenigen Speziallabors verfügbare umfassende Diagnostik ist in Tab. 2 dargestellt. Unabdingbar ist hier, dass Blutproben möglichst zügig zu Serum und EDTA-Plasma verarbeitet werden und - sofern die Analyse nicht sofort möglich ist - das Probenmaterial tiefgefroren wird und der Versand in die Speziallabors auf Trockeneis per Courier erfolgt. Während für die Analyse der Gesamtfunktionen (CH50 und AH50), der Komplementproteine und -regulatoren sowie der Autoantikörper Serum ausreicht bzw. erforderlich (Funktionstest für den C3-Nephritisfaktor) ist, ist eine Quantifizierung von Aktivierungsprodukten nur im EDTA-Plasma möglich. Die Erstuntersuchung sollte immer die globale Funktionsbestimmung des gesamten Komplementsystems (CH50, AH50) und die Analyse eines Aktivierungsprodukts beinhalten.

\section{Therapie komplementvermittelter Erkrankungen}

Während es, abgesehen von möglichen temporären Plasmagaben, mit Ausnahme des C1-Inhibitordefektes keine spezifische Substitutionstherapie für einen Komplementdefekt gibt, hat die Behandlung komplementvermittelter inflammatorischer Erkrankungen in den letzten Jahren große Fortschritte gemacht. Der pathophysiologischen Bedeutung des Anaphylatoxins C5a und des Membranangriffskomplexes Rechnung tragend, hat sich das Komplementtargeting bisher im Wesentlichen auf die Blockade von C5 konzentriert. Der humanisierte Anti-C5-Antiköper Eculizumab ist zurzeit für die Indikation $\mathrm{PNH}$, aHUS und generalisierte Myasthenia gravis zugelassen, wird aber bereits seit Jahren bei einer Vielzahl anderer Krankheitsbilder (wie C3G, SLE-Nephritis, AMD, akute humorale Transplantatabstoßung der Niere) im individuellen Heilversuch eingesetzt. Die dadurch bewirkte Blockade der terminalen Sequenz der Komplementkaskade gewährleistet die sehr wichtige Abwehrfunktion der Opsonsierung, nimmt aber keinen Einfluss auf die Aktivierungsphase, was für die komple-

Tab. 2 Komplementdiagnostik und Indikation

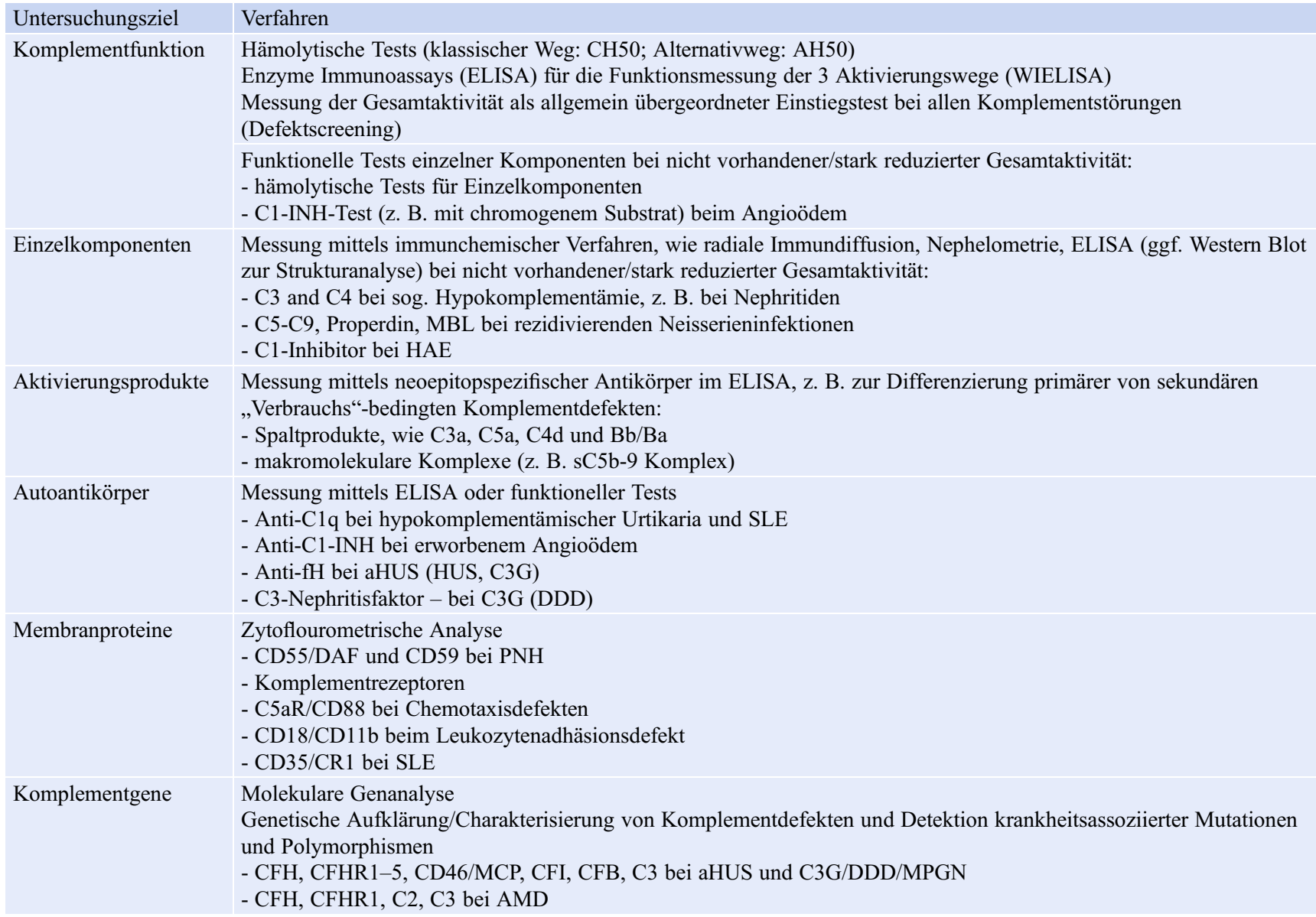

$M B L$ Mannose-bindendes Lektin; HAE hereditäres Angioödem; SLE systemischer Lupus erythematodes; $a H U S$ atypisches hämolytisch-urämisches Syndrom; $P N H$ paroxysmale nächtliche Hämoglobinurie 
mentvermittelte Opsonisierung von Pathogenen bedeutend, aber auch für das Verständnis der Komplementanalytik im Monitoring der behandelten Patienten wichtig ist. Mit stark erweiterter Indikation laufen zurzeit viele an verschiedenen Stellen des Komplementsystems (C3, Faktor D, Properdin, C5a-Rezeptor) ansetzende Therapiestudien in Phase 2 und 3, sodass zu erwarten ist, dass mit einem differenzierteren Targeting der Komplementkaskade die Behandlung eines großen Spektrums komplementvermittelter Erkrankungen möglich wird.

\section{Weiterführende Literatur}

Al-Herz W, Bousfiha A, Casanova JL et al (2011) Primary immunodeficiency diseases: an update on the classification from the international union of immunological societies expert committee for primary immunodeficiency. Front Immunol 2(54):12

Botto M, Kirschfink M, Macor P, Pickering MC (2009) Complement in human diseases: Lessons from complement deficiencies. Mol Immunol 46(14):27744):27

Cicardi M, Aberer W, Banerji A, Bas M, Bernstein JA, Bork K, Caballero T, Farkas H, Grumach A, Kaplan AP, Riedl MA, Triggiani M, Zanichelli A, Zuraw B, HAWK under the patronage of EAACI (European Academy of Allergy and Clinical Immunology) (2014) Classification, diagnosis, and approach to treatment for angioedema: consensus report from the hereditary angioedema international working group. Allergy 69:602-616

Degn SE, Jensenius JC, Thiel S (2011) Disease-causing mutations in genes of the complement system. Am J Hum Genet 88(6):689

Farkas H, Martinez-Saguer I, Bork K, Bowen T, Craig T, Frank M, Germenis AE, Grumach AS, Luczay A, Varga L, Zanichelli A, HAWK (2017) International consensus on the diagnosis and management of pediatric patients with hereditary angioedema with C1 inhibitor deficiency. Allergy 72(2):300-313

Grumach AS, Kirschfink M (2014) Are complement deficiencies really rare? Overview on prevalence, clinical importance and modern diagnostic approach. Mol Immunol 61:110-117

Hajishengallis G, Reis ES, Mastellos DC, Ricklin D, Lambris JD (2017) Novel mechanisms and functions of complement. Nat Immunol 18(12):1288-1298

López-Lera A, Corvillo F, Nozal P, Regueiro JR, Sánchez-Corral P, López-Trascasa M (2018) Complement as a diagnostic tool in immunopathology. Semin Cell Dev Biol 85:86-97

Maurer M, Magerl M, Ansotegui I, Aygören Pürsün E, Betschel S, Bork K, Bowen T, Balle Boysen H, Farkas H, Grumach A,
Hide M, Katelaris C, Lockey R, Longhurst H, Lumry W, MartinezSaguer I, Moldovan D, Nast A, Pawankar R, Potter P, Riedl M, Ritchie B, Rosenwasser L, Sánchez-Borges M, Zhi Y, Zuraw B, Craig $\mathrm{T}$ (2018) The international WAO/EAACI guideline for the management of hereditary angioedema - the 2017 revision and update. Allergy 73(8):1571-1754.

Mayilyan KR (2012) Complement genetics, deficiencies, and disease associations. Protein Cell 3(7):487-496

Merle NS, Church SE, Fremeaux-Bacchi V, Roumenina LT (2015a) Complement system part I - molecular mechanisms of activation and regulation. Front Immunol 6:262

Merle NS, Noe R, Halbwachs-Mecarelli L, Fremeaux-Bacchi V, Roumenina LT (2015b) Complement system part II: role in immunity. Front Immunol 6:257

Pettigrew HD, Teuber SS, Gershwin ME (2009) Clinical significance of complement deficiencies. Ann N Y Acad Sci 1173:108-123.

Picard C, Bobby Gaspar H, Al-Herz W, Bousfiha A, Casanova JL, Chatila T, Crow YJ, Cunningham-Rundles C, Etzioni A, Franco JL, Holland SM, Klein C, Morio T, Ochs HD, Oksenhendler E, Puck J, Tang MLK, Tangye SG, Torgerson TR, Sullivan KE (2018) International union of immunological societies: 2017 primary immunodeficiency diseases committee report on inborn errors of immunity. J Clin Immunol 38(1):96-128

Ricklin D, Hajishengallis G, Yang K, Lambris JD (2010) Complement: a key system for immune surveillance and homeostasis. Nat Immunol 11(9):785-797

Ricklin D, Reis ES, Lambris JD (2016) Complement in disease: a defence system turning offensive. Nat Rev Nephrol 12(7):383-401

Ricklin D, Mastellos DC, Reis ES, Lambris JD (2018) The renaissance of complement therapeutics. Nat Rev Nephrol 14(1):26-47

Skattum L, van Deuren M, van der Poll T, Truedsson L (2011) Complement deficiency states and associated infections. Mol Immunol 48(14):1643-1655

Vries Ede, European Society for Immunodeficiencies (ESID) members (2012) Patient-centred screening for primary immunodeficiency, a multi-stage diagnostic protocol designed for non-immunologists: 2011 update. Clin Exp Immunol 167(1):108-119

Wahn V, Späth P (2008) Komplementdefekte Kinder- und Jugendmedizin 3:179-184

Wehling C, Amon O, Bommer M, Hoppe B, Kentouche K, Schalk G, Weimer R, Wiesener M, Hohenstein B, Tönshoff B, Büscher R, Fehrenbach H, Gök ÖN, Kirschfink M (2017) Monitoring of complement activation biomarkers and eculizumab in complementmediated renal disorders. Clin Exp Immunol 187(2):304-315

www.ESID.org

www.immundefekt.de 